\title{
APUNTES PARA UNA TEORTA DE LA RAZÓN
}

\section{La revolución lógica y el horizonte filosófico}

Cuando en 1897 Burali-Forti publicó la primera paradoja de la teoría de los conjuntos, ni él ni los que en aquella época se interesaban por el fundamento filosófico de las matemáticas y de la ciencia, sospecharon la trascendencia de descubrimiento. La repercusión fue muy grande en los círculos especializados, pero se consideró que se trataba de un problema local, susceptible de una solución dentro de las posibilidades existentes. La primera consecuencia fue la intensificación del moderno movimiento lógico. Desde hacía ya medio siglo la lógica se estaba desarrollando a velocidad creciente, pero el descubrimiento de las paradojas mostró que el aparato lógico disponible era insuficiente para abordar el problema de acuerdo con sus exigencias intrínsecas. Los esfuerzos se concentraron en la elaboración de un instrumento lógico adecuado para tratar el problema de las paradojas y en general de la derivación matemática. El resultado de este proceso fue descubrir que las posibilidades de desarrollo de la lógica eran muy superiores a todo lo previsto. Pero el desarrollo del instrumento lógico, al aumentar el poder de análisis, abrió una serie de inesperadas perspectivas. El resultado más importante fue que la solución de las paradojas no era un problema tan sencillo ni tan limitado como pudo parecer en los primeros momentos. Pronto se vio que el ataque del problema inicial planteaba nuevos problemas cada vez más amplios, que repercutían sobre todo el ámbito de la lógica y de la fundamentación de las matemáticas. Estos nuevos problemas exigian cada vez mayor eficiencia de las técnicas de análisis y esto obligaba a elaborar sistemas deductivos cada vez más perfectos y amplios. La exigencia de perfección analítica se hizo tan fuerte que fue inevitable crear una nueva técnica para analizar y justificar el propio instrumento lógico. Nace así la metateoría, primero como sintaxis y luego como semántica. Esta nueva técnica analítica abre horizontes mucho más amplios y renovadores que los que, pocos años antes, había abierto la reelaboración lógica. Se llega a conclusiones totalmente inesperadas sobre el problema de las paradojas y de los fundamentos, se descubren relaciones extraordinariamente profundas entre los métodos metateóricos y los sistemas matemáticos y se abren perspectivas revolucionarias sobre el significado de la lógica y del conocimiento matemático. Aunque en este gigantesco desarrollo se encuentran los más variados temas, el impulso principal es el desarrollo de la lógica como técnica de análisis para fundamentar el conocimiento cientí- 
fico. Por eso puede considerarse todo el movimiento como una revolución lógica, como una ruptura de los moldes tradicionales del pensamiento deduc. tivo y como la consecuente revelación de nuevas posibilidades en la construc. ción y manejo de las teorías exactas.

El impacto de la revolución lógica sobre la concepción filosófica de las matemáticas ha sido dramático y su importancia es hoy día universalmente reconocida. Pero no se ven aún con la misma claridad las consecuencias que este impacto tiene para la temática filosófica general. Sin embargo, estas consecuencias son aún de mayor importancia que las anteriores, porque abarcan un campo no sólo más amplio sino más fundamental. Si la lógica es algo diferente de lo que se creía, si los principios que rigen el conocimiento deductivo son más amplios que los tradicionalmente considerados, entonces es necesario revisar toda la historia de la filosofía. Porque la filosofía ha pretendido justificar sus tesis racionalmente y para ello se ha valido siempre, de manera explícita o implícita, de la lógica. La concepción de la lógica de los diversos sistemas filosóficos ha presupuesto siempre una determinada concepción de la razón, y la concepción de la razón ha sido el fundamento último de la posibilidad filosófica. Pero no sólo la renovación de la lógica formal repercute sobre toda la filosofía. Los desarrollos de la técnica analítica que culminan en el advenimiento de la metateoría, desembocan en un nuevo planteamiento de la teoría de la verdad, profundamente vinculada con la teoría general de la razón. Se trata en realidad de aspectos de un mismo planteamiento: la fundamentación última de todo conocimiento como tal.

La revolución lógica que se inicia como intento de resolver un problema científicamente localizado, se desarrolla hasta envolver en su poderoso torbellino el corazón mismo de la filosofía. Su verdadera importancia reside por eso más que en los resultados alcanzados por la moderna filosofía de las matemáticas -algunos de ellos asombrosos_ en su repercusión filosófica. Esta repercusión no es parcial sino total y exige por eso el replanteamiento de los grandes temas clásicos desde una perspectiva diferente. El replanteamiento a su vez desemboca en el descubrimiento de una problemática especifica, característica del momento filosófico, cuyo tratamiento habrá de marcar la pauta para una futura reestructuración filosófica.

En las líneas que siguen intentamos mostrar, de la manera más resumida posible, la trayectoria que lleva de la revolución lógica al replanteamiento y reestructuración de la gran temática filosófica. No pretendemos de ninguna manera ofrecer soluciones. Lo que interesa es mostrar el sentido profundo del proceso. La reestructuración final sólo podrá lograrse una vez que se haya alcanzado la plena comprensión de las razones que obligan a plantear su necesidad.1

1 La mayoría de los desarrollos que siguen son reelaboraciones (algunas de ellas bastante amplias) de trabajos presentados en diversos congresos interamericanos de filosofía y 


\section{El concepto de razón y el rebasamiento de las doctrinas tradicionales}

a) Los limites de los formalismos. El resultado más importante y sensacional de todos los obtenidos en el proceso descrito, que constituye un verdadero punto de partida en el camino que conduce a resultados cada vez más imprevistos, es el descubrimiento de que el lenguaje formal, medio expresivo esencial al pensamiento matemático, no puede agotar dicho pensamiento. El famoso teorema de incompleción de Kurt Gödel, demostrado en 1931, reveló algo totalmente inesperado: la insuficiencia de la forma expresiva de la matemática ante el contenido objetivo. Esto obligó a cambiar todas las expectativas sobre el conocimiento matemático.

Clásicamente se creyó siempre que todos los problemas matemáticos eran, en principio, solubles. Se tuvo la convicción de que, positiva o negativamente, era posible decidir la validez de una proposición matemática. La solución negativa de una serie de problemas clásicos, como la cuadratura del círculo o la tripartición del ángulo, reforzó la creencia en la decidibilidad fundamental de todo problema. Las matemáticas eran consideradas, por este motivo, como la ciencia más perfecta, en que la razón podía abarcar todas las posibilidades y esclarecer todas las oscuridades, aplicando sus principios evidentes e inmutables. La dificultad en la solución de los problemas radicaba sólo en las limitaciones psicológicas individuales. Pero con el suficiente ingenio era posible resolver - positiva o negativamente_ cualquier problema. En principio la razón matemática no tenía límites.

Empero, el descubrimiento de las paradojas mostró que los principios racionales que se habian empleado en la demostración de los teoremas clásicos, no eran tan evidentes ni tan inconmovibles. Más aún, existía una enorme vaguedad y confusión en el pensamiento de los más ilustres matemáticos cuando se trataba de los principios racionales que se empleaban en la derivación de los teoremas. La única manera de luchar contra las paradojas y de encontrar un modo, no sólo de suprimir las existentes sino de ofrecer una garantía contra las futuras, era esclarecer rigurosamente el concepto de principio lógico y de derivación matemática. Pero la única manera de hacer esto era mediante un adecuado proceso de formalización, es decir, mediante un lenguaje que fuera capaz de expresar de manera completa todos los conceptos empleados en las definiciones, todas las reglas utilizadas en la formación de las proposiciones matemáticas, todos los postulados $\mathrm{y}$ todos los principios lógicos empleados en la derivación de los teoremas. Mientras esto no se hiciera, el proceso demostrativo seguiría siendo un proceso vago, con el consecuente peligro de producción de paradojas. La formalización fue, pues, una consecuencia natural e inevitable del afán de superar las paradojas. Por otra parte,

en sesiones de la Sociedad Peruana de Filosofía. Las tesis sobre el lenguaje y la expresión filosófica son de elaboración reciente. 
el lenguaje matemático ingenuo, comparado con el de otras ciencias, es el más formalizado. Todo matemático tiene la clara conciencia de que sólo a través de la formalización puede lograrse el rigor necesario a su ciencia. Por eso el implacable proceso de formalización del lenguaje matemático que originó el descubrimiento de las paradojas, fue visto como un proceso natural, como la culminación de la evolución del conocimiento matemático hacia niveles de rigor cada vez más elevados.

Cuando, después de no pocos esfuerzos y de no poca dosis de genio, los protagonistas de la revolución lógica lograron formalizar la aritmética y probaron que en principio cualquier disciplina matemática podía formalizarse, se creyó que se había logrado rigorizar por completo la ciencia matemática. Naturalmente, en la nueva aritmética formalizada, se pudieron probar todos los teoremas clásicos y se llegó por eso a la conclusión de que la expresión formalizada era la culminación de la ciencia matemática. A través de los desarrollos formales, la razón, gracias a sus principios supremos, podía alcanzar el conocimiento cabal de su objeto. Por otra parte, una vez convenientemente formalizados los diversos sistemas matemáticos, era posible estudiar sus propiedades formales y determinar si dichos sistemas eran o no susceptibles de engendrar paradojas. Las primeras investigaciones mostraron que gran parte de la aritmética era consistente, es decir, que convenientemente formalizada, no podía originar paradojas. La razón no sólo podía alcanzar un conocimiento completo de su objeto en el campo matemático, sino que además podía liberarse de contradicciones internas. Se había llegado a un estado que permitia justificar plena y cabalmente todas las expectativas del racionalismo.

El teorema de Gödel derrumbó todas estas esperanzas. Porque mostró que cuando se trataba de la aritmética clásica (no de aspectos parciales del sistema sino de todo el sistema tal como se determina mediante los postulados de Peano) era imposible demostrar la consistencia de la aritmética por métodos absolutamente seguros, y además, que el formalismo creado para la aritmética, que parecía un lenguaje perfecto, no era suficiente para derivar todos los teoremas posibles. Kurt Gödel probó que era posible construir una proposición aritmética, con las reglas permitidas en el sistema formal, de manera que ni ella ni su negación podían derivarse dentro del sistema. Probó además que, desde un punto de vista intuitivo, dicha proposición era verdadera. Quedó así establecido que cuando se expresa una teoría matemática mediante un lenguaje completamente formalizado, existen proposiciones verdaderas de la teoría, que no pueden derivarse como teoremas dentro del lenguaje formalizado. $O$, lo que es lo mismo, probó que el objeto del conocimiento matemático rebasa la esfera del lenguaje matemático, que la matemática no puede confundirse con el lenguaje que la expresa, porque, por 
más perfecto que sea un lenguaje, nunca podrá utilizarse para derivar todos los posibles teoremas de la teoría que formaliza. ${ }^{2}$

La situación se tornó altamente desconcertante. De un lado, el enfoque intuitivo de la matemática llevó a la aparición de las paradojas. Para eliminar las paradojas fue necesario formalizar la matemática, es decir, crear lenguajes formales capaces de expresar explícitamente todos los elementos del conocimiento matemático. Una vez lograda la formalización se desarrolló un método (metateoría) para mostrar, de manera irrefutable, que era posible construir sistemas formalizados consistentes. Pero el resultado fue que no podía obtenerse esta demostración de manera irrefutable y que la formalización no agotaba la riqueza del conocimiento matemático. El análisis del proceso de formalización que se originó en el afán de evitar las vaguedades intuitivas de la matemática que condujeron a las paradojas, obligó a reconocer que la matemática intuitiva no podía ser eliminada por medio de ningún lenguaje formalizado.

La situación era inesperada. Pero no era un mero retorno al punto de partida. Entre la matemática intuitiva que originó las paradojas y el residuo intuitivo que rebasaba la formalización realizada, existía una creación metodológica de excepcional penetración. Por eso este impasse constituyó el punto de partida de nuevas investigaciones y de ulteriores y cada vez más profundos esclarecimientos. Pero el resultado de Kurt Gödel, por sí solo, constituye un callejón sin salida para el pensamiento convencional. Es en sí el primer e inevitable obstáculo que obliga a la renovación de ciertas maneras tradicionales de pensar.

b) La insuficiencia de los sistemas tradicionales. La consecuencia filosófica más extraordinaria de la demostración gödeliana es la pérdida de vigencia del principio del tercio excluido. Todo el pensamiento matemático clásico se había desenvuelto sobre el presupuesto del "tertium non datur", y es la vigencia indiscutida de este principio lógico lo que constituyó el fundamento de la creencia ingenua de que todo problema matemático es soluble positiva o negativamente. Pero, en la aritmética formalizada, es siempre posible construir proposiciones tales que, tanto ellas como sus negaciones son inderivables. $O$ sea, que en relación al sistema formal dichas proposiciones no pueden ser ni verdaderas ni falsas, porque, matemáticamente, la verdad

2 Cuando se inició el movimiento formalista, muchos pensadores, especialmente los in. tuicionistas, sostuvieron que el ideal formalista era inalcanzable. Pero no pudieron probarlo. Sólo Kurt Gödel pudo dar una prueba de la limitación enunciada. Pero si los formalistas hubieran tenido éxito, si hubieran podido construir un lenguaje que permitiese: $r$ ) probar que las matemáticas son consistentes, y 2) que existe un lenguaje que permite expresar y derivar todas las verdades matemáticas, se habría llegado a la conclusión de que la esencia de las matemáticas reside en su expresión lingüística y que el conocimiento matemático es racionalmente perfecto. 
o falsedad de toda proposición que no sea un postulado sólo puede determinarse por medio de la derivación o prueba.

Puede pensarse que esto sucede sólo en relación al sistema formalizado, pero que en relación a la matemática intuitiva, tal como se constituye en la investigación clásica, el principio conserva su vigencia. Mas quien comprende lo que significa el teorema de Kurt Gödel, no puede hacer esta afirmación, porque si la matemática no se formaliza y se mantiene en el nivel intuitivo clásico, entonces se producen las paradojas o, en el mejor de los casos, desaparecen todas las garantías contra ellas. Estamos pues ante un verdadero dilema: o se conserva el principio del tercio en la matemática intuitiva y se produce el peligro de encontrar la contradicción en el fundamento mismo del pensamiento matemático, o se formalizan los sistemas matemáticos y el principio del tercio excluido no puede mantener su validez absoluta.

Esta pérdida de vigencia de un principio lógico considerado fundamental es uno de los fenómenos más sorprendentes de la historia de la filosofía, porque se trata de una evolución interna del pensamiento que conduce a consecuencias inevitables. No se trata de un cambio de perspectiva histórica, no se trata de un cambio en los valores o en las jerarquías, del triunfo de una escuela sobre otra. Se trata de algo muy diferente: de un resultado lógico, obtenido dentro de las técnicas más rigurosas de la misma lógica. Por otra parte, todas las escuelas habian aceptado siempre su validez, aunque discreparan en la manera de interpretälo y sobre todo de fundamentarlo. ${ }^{3}$

I. El racionalismo que, a pesar de haber perdido su viejo predominio, se mantenía aún vigoroso en movimientos como la fenomenología, recibe su golpe de gracia. Porque a través de toda la tradición racionalista se ha hablado de los principios supremos del pensamiento y el principio del tercio ha sido considerado uno de estos principios. No puede concebirse el racionalismo sin la existencia de un sistema de principios captados por medio de una evidencia intelectual indubitable. El advenimiento de las geometrías no euclidianas y el impacto producido por la teoría de la relatividad y sobre todo, por la teoría de los quanta, había ya mostrado las dificultades insuperables del racionalismo. Pero la pérdida de vigencia de uno de los principios fundamentales de la razón es el derrumbe definitivo. Después de la revolución lógica no puede hablarse más de una razón concebida como una estructura cerrada de principios evidentes.

II. El empirismo no puede tampoco dar cuenta de la situación. La revolución lógica había obligado a los empiristas a reconocer que los principios

3 Incluso los mismos hegelianos y marxistas deben aceptar que, en el campo de los enunciados, deben respetarse los principios lógicos formales y que no tiene sentido enunciar dos proposiciones contradictorias o una proposición que no sea ni verdadera ni falsa. Las modernas tendencias de la lógica dialéctica, tanto en Occidente (por ejempló, Lefevbre) como en la Unión Soviética aceptan la validez de los principios lógicos tradicionales. 
lógicos no pueden derivarse de la experiencia. Para evitar el dogma racionalista adoptaron una posición convencionalista. Los principios lógicos son sólo reglas del lenguaje para expresar los fenómenos y estas reglas son convencionales. Las matemáticas no son sino la prolongación de la lógica. Los resultados de Gödel invalidan esta posición. En primer lugar la pérdida de vigencia del tertium no se deriva de ningún hecho empírico. Se deriva de una necesidad del pensamiento lógico y matemático, de una evolución interna del pensamiento racional que parece mostrar que existe algo así como una región independiente de la experiencia con un dinamismo propio. No una razón terminada y arquitectónica, cuyos principios son evidentes, pero sí una razón que impone una necesidad y que no puede reducirse a puras reglas convencionales. En segundo lugar, y esto es definitivo, el descubrimiento de los límites de los lenguajes formales muestra que las matemáticas no pueden reducirse al lenguaje sino que lo rebasan. Existe pues un mundo matemático que no es empírico, pero que no puede reducirse a la categoría de lenguaje convencional. No hay salida.

III. El pragmatismo no ofrece una mejor posibilidad. De acuerdo con su tesis fundamental, los resultados descritos deberían poderse comprender en relación con la eficacia de la acción. Pero el profundo proceso que conduce a los sorprendentes resultados de Gödel no tiene nada que ver con la acción. El proceso que conduce a las paradojas y al intento de ofrecer garantías contra ellas, es un proceso interno del pensamiento. En ningún momento la urgencia de la acción ha orientado el movimiento. La revelación final de que el lenguaje no puede agotar el contenido de las matemáticas, de que existe un residuo intuitivo en el pensamiento matemático del cual no nos podemos librar y que nos remite a un tipo muy especial de intuición intelectual, no tiene nada que ver con la eficacia de la acción. Todo el proceso es el resultado de un movimiento interno del pensamiento que culmina en una serie de resultados inevitables, que se imponen por una indiscutible necesidad racional.

Pero hay más todavía. El principio del tercio excluido ha demostrado a través de la historia su extraordinaria eficacia para la acción. No sólo el pensamiento científico clásico y el pensamiento técnico, sino hasta la acción política lo han empleado de manera ininterrumpida y con éxito indiscutible. $Y$, sin embargo, por un proceso totalmente abstracto del pensamiento alejado de la realidad, y sin ninguna repercusión práctica, se ha tenido que limitar en su universalidad.4

4 Podría pensarse que la utilidad del principio del tercio para la acción es sólo en relación con el mundo físico, en regiones finitas del universo, y en consecuencia, en relación a conjuntos finitos de objetos. Pero el principio del tercio excluido es también útil para la demostración de teoremas de la teoría de los conjuntos y del análisis, es decir, en relación a la determinación de propiedades de conjuntos infinitos. Ciertos teoremas fundamentales del análisis no pueden demostrarse sin su ayuda, y el análisis es el instrumento fundamen- 
IV. El materialismo no es una escuela comparable a las anteriores, pues se trata de una posición que se define mediante una tesis de carácter concreto: todo en el universo es material, el espíritu es un epifenómeno. Aunque por lo general no se hace suficiente hincapié sobre la relación entre el racionalismo y el materialismo, este último no es sino un tipo de doctrina metafísica y, como tal, está clásicamente unida al racionalismo. Pero es interesante referirse al materialismo debido a que una de sus especies, el materialismo dialéctico, ha querido interpretar los resultados de Gödel como una confirmación de los principios dialécticos. ${ }^{\bar{\sigma}}$ Según el materialismo dialéctico los resultados de Gödel, en el sentido de que no puede demostrarse de manera indubitable (como se creyó en un principio que se podría) la consistencia de la aritmética, demuestra que la contradicción es el motor del pensamiento matemático. Nada menos fundado que esta interpretación. El pensamiento matemático jamás ha procedido impulsado por el afán de superar contradicciones, sino por el afán de encontrar nuevas verdades y de resolver problemas que en sí nada tenían de contradictorios, ni contradecían otros aspectos del sistema. En todos sus desarrollos ha presupuesto siempre, como el principio supremo, el principio de no contradicción, y es tal la vigencia de este principio que apenas se descubrieron las paradojas se trató de inmediato de eliminarlas. Se ha podido eliminarlas, y el resultado no ha sido de ninguna manera un sistema en que las paradojas han sido incorporadas como elementos de una síntesis superior. La dificultad de encontrar una garantía definitiva contra ellas no significa que el pensamiento matemático progrese a través de contradicciones. El hecho de que se descubrieran contradicciones que han sido eliminadas (aunque sin una garantía absoluta de que no se descubrirán nuevas) no significa que el pensamiento marche dialécticamente. Que el pensamiento humano se tope frecuentemente con contradicciones y que trate de superarlas es un hecho que se ha reconocido clásicamente. Pero el pensamiento dialéctico no consiste en afirmar esto, sino en la manera como concibe el proceso que genera la existencia de la contradicción y la superación de la misma. Nada de esto puede encontrarse en el pensamiento que se origina en el descubrimiento de las paradojas y que asciende hasta la perspectiva metateórica.

V. La imposibilidad de dar cuenta del proceso que conduce al teorema de Kurt Gödel y a sus resultados por las escuelas clásicas, sólo deja una puerta abierta: el relativismo. El hecho de que durante milenios se haya considerado, no sólo en la filosofia oficial, sino en los presupuestos de la investigación científica, que el principio del tercio excluido tiene valor absoluto

tal de la técnica, es decir, de la trasformación del mundo. La eficacia de la acción presiona; como se ve, en un sentido inverso al del movimiento que ha culminado en Ia pérdida del valor absoluto del tertium.

5 Ver el prologo de Haldane a la edición española de la Filosofia de la Naturaleza de Engels. 
y universal, y que repentinamente se haya demostrado su limitación y haya sido incluso rechazado sistemáticamente por una escuela filosófico-matemática, demuestra, según los relativistas, que los principios racionales son función de la época histórica y que el conocimiento es relativo a las vigencias exigidas por una situación vital determinada.

Fuera de las dificultades insalvables a las que conduce todo relativismo (la imposibilidad de justificar el valor absoluto de la propia tesis), el carácter de la situación hace imposible una salida relativista. Porque dentro del hecho total existe un hecho parcial, que no ha sido suficientemente observado y que invalida $a b$ initio toda interpretación relativista: la pérdida de vigencia absoluta del principio del tercio excluido se debe a la imposibilidad de prescindir del principio de no contradicción. ${ }^{6}$ Es, en efecto, la necesidad de eliminar las paradojas y de ofrecer una garantía contra ellas, lo que genera el hondo proceso que conduce a la limitación de la validez del principio del tercio. Metafóricamente hablando, se sacrifica un principio racional para salvar otro. $\mathrm{Y}$ se hace esto por una necesidad absoluta. En todo el proceso el principio de no contradicción se manifiesta con una evidencia incontrastable. EI proceso, en lugar de mostrar que los principios racionales son relativos, muestra que existe un principio absoluto, el principio antifásico, cuya vigencia inconmovible obliga a limitar otro principio que también se creía absoluto. De acuerdo con la estructura constitutiva de la lógica, si en un sistema deductivo puede demostrarse una contradicción, entonces puede demostrarse cualquier proposición construible dentro del sistema. $O$ sea, que la existencia de una contradicción deductivamente fundada (o, por supuesto, puramente postulacional), anula ipso facto el sistema. Esta consecuencia es inescapable, es una propiedad estructural de todo sistema deductivo, es una exigencia permanente del pensamiento. Todo hace pensar que se trata de una vigencia suprahistórica de la razón.

La situación es por cierto desconcertante, tan desconcertante que, como acabamos de ver, rebasa las posibilidades de los planteamientos clásicos. $\mathrm{Y}$, sin embargo, debe ser enfocada e interpretada de algún modo, pues se trata de una temática que incide profunda y esencialmente sobre los problemas fundamentales del pensamiento tradicional. 7

6 En 1954, hice por primera vez referencia a este hecho que considero fundamental, en un ciclo de conferencias en el Instituto Riva Agïero de la Universidad Católica de Lima sobre "Lógicas no aristotélicas". En 1956, en un trabajo aparecido en la Revue de Methaphysique et de Morale, "Crise de la science et théorie de la raison" me refiero al proceso que hemos descrito en las líneas que anteceden y hago hincapié sobre el hecho mencionado.

7 Lo dicho vale para todo sistema de lógica, incluso para aquellos, como el de Fitch, en los que se considera, además de proposiciones verdaderas y falsas, proposiciones de un tercer valor (indefinidas). En este tipo de sistemas no tiene vigencia el principio del tercio, pero el principio de no contradicción tiene el mismo valor que en los sistemas; si no fuera 
c) Hacia una teoria de la razón. La imposibilidad de las escuelas tradicionales de dar cuenta de la situación originada por el proceso de la revolución lógica muestra la necesidad de replantear y reelaborar importantes temas clásicos. Si se interpretan adecuadamente el significado de estos temas y la relación que existe entre ellos -evidencia de los principios racionales, necesidad de limitar la vigencia de uno de ellos para mantener la vigencia de otro, limitación de los sistemas formales para expresar todas las posibilidades intuitivas, descubrimiento de un residuo intuitivo- se ve que todos convergen hacia un núcleo central: el tema de la razón. En efecto, el problema de la intuición intelectual, de los principios, de las posibilidades expresivas del lenguaje, sólo tienen sentido en relación a la actividad fundamental del conocimiento racional. El interés de todos los problemas revelados a través del anterior análisis, reside en que inciden directamente en el problema del conocimiento científico. Basta este hecho para que el tema de la razón aparezca en el fondo de todos los problemas. Pero el hecho de tratarse del conocimiento matemático, muestra que se trata del conocimiento racional por excelencia. $\mathrm{Y}$ esto refuerza e intensifica la convergencia problemática hacia el tema central de la razón.

El resultado más importante del análisis efectuado es que, aunque las posiciones clásicas son incapaces de ofrecer una comprensión adecuada del proceso descrito, ello no significa de ninguna manera que se trate de algo caótico o sin sentido. Hemos visto que se trata de un movimiento que sigue pautas propias, de un dinamismo de desarrollo intrínseco que conduce inexorablemente hacia ciertos resultados. Estos resultados son desconcertantes en tanto rebasan los moldes clásicos y las costumbres inveteradas de pensar. Pero este rebasamiento y este desconcierto se deben precisamente a la necesidad interna del proceso. Todos nos obliga a aceptar que existe una dinámica de la razón, una manera de funcionar del pensamiento racional que, debido a su profundidad y a la falta de medios analíticos adecuados, ha escapado a la comprensión filosófica.

La única manera, por eso, de comprender los fenómenos descubiertos, es elevarse desde ellos hasta una visión general de la razón, para poder descubrir la unidad y el sentido de todo el proceso. La necesidad de una teoría de la razón es evidente. ${ }^{8}$ Se trata de una tarea filosófica que no puede evitarse porque constituye el campo de exploración impuesto por la dinámica de la marcha del conocimiento racional: se trata de una reelaboración de la teoría de la razón.

asi, a todo teorema podría oponerse su contradictorio, lo que reduciría el sistema a un conjunto sin ningún valor.

8 No debemos olvidar que la única manera de obtener esta demostración es mediante la formalización de la teoría cuya consistencia se quiere establecer. 


\section{El problema de la razón y el replanteamiento de la temática clásica}

a) La intuición intelectual. El tema de la razón es el punto de convergencia de un complejo temático que comienza con el replanteamiento de ciertos temas fundamentales del pensamiento clásico. En lo que sigue exponemos, de manera sucinta, los primeros resultados de este replanteamiento que parte de temas específicos, derivados de la situación descrita, y que culmina en una toma de posición general sobre la estructura y el dinamismo de la razón.

El tema que se impone en el punto de partida es el de la intuición intelectual, no sólo porque la mayor parte de las teorías tradicionales sobre la razón han girado en torno de este tema sino porque el proceso que analizamos se desenvuelve sobre la base de la relación entre la matemática intuitiva y la matemática formalizada. Como hemos visto, el origen del proceso es la necesidad de superar las paradojas derivadas de una metodología basada en la utilización ingenua de ciertas verdades matemáticas y de determinados principios lógicos. Y el punto de llegada es la comprobación de que la formalización, que es el procedimiento metodológico necesario para la eliminación principista de las paradojas, no puede agotar la verdad intuitiva de las matemáticas. Todo el proceso se desarrolla como una lucha entre la necesidad de formalizar y la imposibilidad de librarse de conocimientos intelectuales intuitivos. Por eso, mientras no se esclarezca cuál es el verdadero sentido de estos conocimientos y de la imposibilidad de eliminarlos, no puede comprenderse la temática restante, que, en todo momento, la presupone.

El primer resultado que se desprende del análisis, es que, sin ninguna duda, los criterios clásicos para determinar la existencia de evidencias absolutas, no pueden ya mantenerse: Pero esto no significa que debamos rechazar la existencia de una intuición intelectual y de una evidencia correspondiente. Lo que no puede negarse es que el complejo de evidencias racionales que constituyó la base del racionalismo clásico, se ha disuelto. Pero lo que no ha sido debidamente realzado es que no han desaparecido todas las evidencias. Algunas de las evidencias del complejo, como la del principio antifásico, se han mantenido, mientras que otras han desaparecido, como la del principio del tercio. Se trata de un fenómeno sui generis, muy diferente de otros procesos disolutivos, como, por ejemplo, el de la evidencia geométrica. El advenimiento de las geometrías no euclidianas mostró que no puede hablarse de evidencia geométrica. No hay ninguna intuición indubitable de cualidades o relaciones entre entes que puedan ser llamados geométricos.

Pero esto no significa que todas las disoluciones de evidencias deban ser del mismo tipo. La disolución de las evidencias lógicas es de tipo muy diferente. Porque uno de los principios ha permanecido inconmovible. Es posible rehacer la geometría prescindiendo de cualquiera de los axiomas eucli- 
dianos, puesto que todos son independientes. Pero no es posible rehacer la lógica prescindiendo de cualquiera de sus principios. El principio del tercio y el de no contradicción son independientes, puesto que es posible rehacer la lógica prescindiendo del primero. Pero a pesar de su independencia no puede prescindirse del segundo.9

El análisis del proceso nos pone así en el cauce que debe seguirse. Existe una intuición intelectual, pero es diferente de lo que siempre ha creido el pensamiento racionalista. Esta diferencia se deriva del hecho de que la constelación de evidencias lógicas que siempre aceptó el racionalismo se ha desgranado.

El proceso de desgranamiento nos conduce a su vez a un problema que puede considerarse, en relación a la situación existente, el problema fundamental de la intuición intelectual: si existe un proceso histórico según el cual ciertas evidencias universalmente aceptadas durante un determinado periodo permanecen, mientras otras pierden su vigencia, ¿cuál es el criterio que permite distinguir las evidencias permanentes de las transitorias? El hallazgo de este criterio habrá de constituir, sin duda, la solución, o, por lo menos, el encauzamiento adecuado del análisis.

Hemos visto que en el término del proceso de formalización necesario para probar la consistencia de las matemáticas se descubre un residuo intuitivo que la formalización no puede agotar, es decir, se descubren verdades matemáticas intuitivas que no pueden enmarcarse dentro del sistema formalizado. Ahora bien, antes de la formalización, en la teoría matemática intuitiva, tal como se presenta clásicamente, se aplican determinados principios lógicos, que son tomados por el científico como evidencias absolutas. Cuando se formaliza la teoría intuitiva, se incluyen en la formalización todos los principios lógicos que sirven de base a dicha teoría. Pero una vez que se analiza metateóricamente la teoría formalizada se descubre que algunos de estos principios no tienen el valor absoluto que les reconocía la teoria intuitiva. La teoría formalizada ha servido, pues, como cedazo, como filtro, de los principios de la teoría intuitiva. Una vez que nos convencemos de que ciertos principios que la teoría intuitiva aceptaba como absolutos tienen una aplicación limitada, tenemos que pensar que no hay ninguna razón para seguir usándolos en la teoría intuitiva. Porque si los seguimos usando, estamos rebasando su campo de aplicación. Si no tienen valor universal y los aplicamos universalmente, podemos demostrar una serie de teoremas sin verdadero fundamento (puesto que en el desarrollo intuitivo no existe el control insobrepasable del análisis de las posibilidades derivativas). Pero si en la teoría intuitiva no pueden reconstruirse todos los teoremas prescindiendo de estos principios, la situación sería desesperada. Existiría un verdadero

9 Por supuesto, puede partirse de postulados que no sean una expresión directa del principio antifásico, pero de los cuales éste se pueda derivar. 
callejón sin salida: de un lado, formalizando la teoría intuitiva se llega a la conclusión, a través del análisis metateórico, de que un principio no tiene vigencia universal; del otro se impone el empleo universal de este principio como única posibilidad de desarrollar cabalmente la teoría intuitiva. La teoria formalizada no sólo no agotaría a la teoría intuitiva, sino que nos impondria condiciones que no podrían ser verificadas en la intuición. La rigorización obtenida a través de la formalización, no sólo sería incompleta, sería, además, inútil, puesto que las limitaciones descubiertas a través del análisis metateórico, no podrían mantenerse en la intuición.

Felizmente éste no es el caso. La teoría intuitiva cuya formalización lleva a las conclusiones expuestas, es decir, a la demostración de la limitación del principio del tercio, es la aritmética. Y se ha demostrado que es posible derivar todos los teoremas de la aritmética clásica intuitiva, prescindiendo del principio del tercio. O sea, que intuitivamente queda verificada la limitación descubierta por medio del análisis de las propiedades de la teoria formalizada. Existe, como se ve, una relación de adaptabilidad entre la versión formalizada y el desarrollo intuitivo. Esta coincidencia permite pensar que se trata de un posible criterio para determinar la validez de las evidencias intelectuales. Cuando la formalización de una teoría intuitiva conduce al desgranamiento de algún complejo de evidencias tradicionalmente aceptadas, conduce también a una selección: aquellos principios evidentes que conservan su vigencia en el sistema formalizado y que no pueden ser eliminados del residuo intuitivo, poseen una validez absoluta. En cambio, aquellos principios que sufren algún tipo de limitación o eliminación como resultado del proceso de formalización y de los cuales puede prescindirse en el residuo intuitivo, se revelan como puramente relativos, derivados de algún tipo de extrapolación o metabasis que ha formado intuición a través del hábito.

El proceso descrito que nos lleva a vislumbrar un criterio para discriminar el valor de las evidencias, caracteriza la formalización de la aritmética. Si pudiese descubrirse el mismo proceso en otros sistemas formalizados, la generalidad de los sintomas reforzaria el criterio propuesto. Vale la pena por eso hacer algunas consideraciones sobre la situación actual en la formalización de las teorías matemáticas. El proceso de la formalización de la aritmética es de importancia capital porque la aritmética es una teoría base. Al lado de la aritmética sólo hay otra teoría fundamentadora de tanta importancia: la teoría de los conjuntos. Si se analizan los resultados de la formalización en este caso se llega a conclusiones muy interesantes. El resultado del aná. lisis metateórico revela que, como en el caso de la aritmética, el formalismo no agota el contenido intuitivo de la teoría. De acuerdo con el teorema de Kurt Gödel, si un formalismo permite expresar la aritmética es sintácticamente incompleto; y éste es el caso de la teoría de los conjuntos. Si el sistema es incompleto, entonces existen proposiciones sintácticamente indecidi- 
bles. ${ }^{10}$ Luego, el principio del tercio queda, como en el caso de la aritmética formalizada, limitado. Pero esta limitación no puede verificarse intuitivamente, porque hay una serie de teoremas de fundamental importancia en la teoría de los conjuntos, que no pueden demostrarse sin recurrir al principio del tercio. O sea, nos encontremos aquí con el callejón sin salida mencionado anteriormente.

En apariencia, el criterio propuesto fallaría en el caso de la teoría de los conjuntos. Pero, en realidad, sucede algo muy importante, que permite reafirmarlo. Porque en la teoría de los conjuntos no existe un verdadero plano intuitivo, en el cual se desarrolle la teoría. En el caso de la aritmética este plano existe. Nadie duda que hay una teoria intuitiva de la aritmética, que puede desarrollarse sobre la intuición de las propiedades básicas de los números naturales. Pero, en cambio, en la teoría de los conjuntos no existe una intuición o evidencia de las propiedades fundamentales de los conjuntos. El objeto conjunto no se da a la intuición intelectual de la misma manera como se da el objeto número natural. Ambos objetos pertenecen a esferas completamente distintas, por eso mientras hay acuerdo universal sobre lo que significa la teoría intuitiva de los números, no hay no puede haber acuerdo sobre lo que sería una teoría intuitiva de los conjuntos. Se ve, así, que el criterio de la coincidencia entre las posibilidades de la formalización y el residuo intuitivo no se cumple, porque no hay un verdadero residuo intuitivo. En la teoría de los conjuntos el desarrollo intuitivo puede llamarse intuitivo porque no se han expresado de manera explicita (formal) todos los presupuestos de la teoría. Pero no puede llamarse intuitiva porque se base en ciertas intuiciones intelectuales. Lo que sucede es que la expresión "teoría intuitiva" tiene un doble sentido: un primer sentido general, en que se toma la expresión como sinónimo de teoría ingenua, de teoría con presupuestos no analizados, y otro sentido particular en que significa, además de lo anterior, teoría cuyos postulados expresan determinadas evidencias. En este segundo sentido se dice que existe una teoría intuitiva de la aritmética. Pero no puede decirse entonces que existe una teoría intuitiva de los conjuntos. $Y$ esto concuerda perfectamente con el sentido y origen de la teoria. La teoría de los conjuntos nace como intento de expresar matemáticamente las propiedades de las multiplicidades infinitas. $Y$ por principio, el infinito no puede captarse intuitivamente. Por eso, desde su nacimiento, encontró fuertes resistencias entre importantes matemáticos, que criticaban la manera como prescindía de las intuiciones básicas. ${ }^{11}$ Vemos, pues, que en el caso de la

10 Basándose en el teorema de Löwenheim-Skokem, Wang ha mostrado que la teoría de los conjuntos es semánticamente incompleta.

11 No debe creerse que la situación es simple. Es. cierto que no existe una "intuición intelectual" del conjunto infinito y de las relaciones entre conjuntos. Pero es innegable que en la presentación primitiva de la teoría se encuentran una serie de principios y de reglas que son verdaderamente intuitivos. El análisis de estos factores es de importancia para 
teoría de los conjuntos, no puede hablarse de una falla del criterio propuesto, porque no existe un verdadero residuo intuitivo. ${ }^{12} \mathrm{El}$ análisis del proceso de formalización de la teoría de los conjuntos nos permite así precisar el criterio propuesto: la posibilidad de conservar universalmente ciertos principios, (después que han sido limitados otros), en el sistema formalizado y en la teoría intuitiva, significa que los principios son absolutos y que su evidencia es auténticamente intuitiva.

Pero esto nos conduce de inmediato a una nueva altura del recorrido. Porque para que el criterio propuesto tenga eficacia, se requiere que exista un verdadero residuo intuitivo. Esto sucede en el caso de la aritmética. Y significa, por tanto, que debe de existir, además de la intuición de los principios lógicos, una intuición de otro tipo de verdades. Esto se debe a que el residuo intuitivo se determina como una incompleción del formalismo aritmético. La proposición indecidible construida por Gödel, y todas las que se han cons. truido después, son proposiciones que no pueden derivarse (ellas o sus negaciones) dentro del formalismo. Pero lo esencial es que tienen un significado intuitivo, lo que demuestra que hay verdades aritméticas que no pueden derivarse por medio del formalismo. Además de los principios lógicos, encontramos, pues, en el residuo intuitivo, nada menos que la misma aritmética. Hay verdades aritméticas captadas intuitivamente por medio de la intuición intelectual.

Ahora bien, de acuerdo con el criterio propuesto puede pensarse en la coincidencia entre las eliminaciones y las retenciones del sistema formalizado y las posibilidades de desarrollar la teoría intuitiva de acuerdo con estos resultados. Pero esta necesidad no existe, porque en el caso de las intuiciones aritméticas no hay ningún resultado de la teoría formalizada que obligue a limitar o a rechazar algún principio aritmético intuitivo. El criterio propuesto no tiene aplicación en este caso porque se trata de un caso diferente. El criterio sólo se aplica en los casos de limitación de principios, es decir, en los casos en que la formalización conduce a situaciones en que se descubre que algunos de los principios que se creían evidentes no funcionan de acuerdo con lo previsto.

En los casos en que no existe limitación, entonces el criterio válido parece

la exploración del problema de la intuición intelectual. Pero hasta donde llega nuestra información, aún no se ha hecho. Por otra parte, en relación al tema que nos interesa, es decir, a la confrontación entre el sistema formalizado y el residuo intuitivo, no puede hablarse de intuición intelectual, porque se trata de la captación intuitiva de las propiedades de los conjuntos infinitos y esta captación no existe.

12 Este hecho abre un nuevo y hondo problema en relación a la incompleción de la teoría de los conjuntos. Si la incompleción es sintáctica es imposible determinar con precisión el significado de la proposición indecidible. Se trataría de una proposición puramente simbólica. Si es una incompleción semántica el problema se generaliza, pues ello querría decir que se han determinado criterios de validez semántica y esto es precisamente lo que no puede hacerse. 
ser la existencia del propio residuo intuitivo. Cuando, por más esfuerzos que se hagan, se demuestra la imposibilidad de que un formalismo abarque una determinada teoría y como resultado de esta imposibilidad se revela un residuo intuitivo, cuya evidencia se impone a través de significaciones unívocas, entonces existe evidencia intelectual.

Puede pensarse que la limitación del formalismo no está ligada a la existencia de esta intuición. Puede pensarse que es perfectamente posible que el formalismo coincida con el estrato intuitivo y que entonces el criterio propuesto no tiene aplicación. Pero, en este caso hipotético, no podría saberse si la intuición es o no es absoluta. No existiría manera de mostrar que existe una intuición intelectual que no se deriva del hábito psicológico. Si la formalización de la aritmética no hubiera sido incompleta todos los matemáticos habrían adoptado un criterio formalista y habrían considerado que las matemáticas no son sino sistemas formales que pueden construirse arbitrariamente. La semántica sólo habría tenido sentido en el caso de la aplicación de las teorías matemáticas a la realidad. Lo revelador es que el estrato intuitivo no ha podido ser eliminado. Esto significa mucho más que la existencia de una intuición intelectual y nos lleva, como veremos a continuación, al significado último del lenguaje. Pero significa de todas maneras que hay una intuición que rebasa todo esfuerzo de formalización y que esta intuición impone sus evidencias. Hay una coincidencia entre el residuo intuitivo y el contenido de la teoría intuitiva. El residuo intuitivo, la verdad aritmética que se prueba intuitivamente no pertenece a una aritmética diferente. Es una verdad que pertenece al campo de la teoría de los números naturales, es decir, a la teoría que se funda en ciertas intuiciones básicas. La existencia del residuo intuitivo redescubre, ratifica, la evidencia de la intuición aritmética y por eso puede tomarse como criterio de evidencia.

Con lo avanzado se ha recorrido ya algún trecho en el cauce impuesto por el análisis del proceso que conduce a la nueva situación. Se ha mostrado que la nueva situación originada por la revolución lógica no anula la posibilidad de que exista la intuición intelectual basada en evidencias de vigencia necesaria y universal. Se ha mostrado que no pueden aceptarse ingenuamente todas las evidencias tradicionales, pero que, en el caso de disolución que se descubre como un inevitable desarrollo intrínseco, puede encontrarse un criterio de selección. Este criterio se basa en las relaciones entre la teoría formalizada y su correspondiente intuitivo. El criterio indicado se desprende del análisis de sólo dos casos: de la aritmética y de la teoría de los conjuntos (que sirve, de acuerdo a los resultados peculiares de la limitación de su formalismo, para reforzar la aplicación positiva del criterio en la aritmética). Es necesario encontrar nuevos casos que muestren que se trata de un criterio general.

Pero de todas maneras el hecho de que el criterio se funde en estas 
dos teorías básicas muestra que no se trata de una generalización precipitada. $\mathrm{Y}$, aunque no puede aún llegarse a conclusiones definitivas, el planteamiento expuesto muestra la existencia de vías de exploración muy diferentes de las tradicionales.

(Continuará)

Francisco Miró Quesada 